\title{
A Critical Time Window for the Recruitment of Bulbar Newborn Neurons by Olfactory Discrimination Learning
}

\author{
Laure Belnoue, ${ }^{1,2}$ Noelle Grosjean, ${ }^{1,2}$ Djoher Nora Abrous, ${ }^{1,2 *}$ and Muriel Koehl ${ }^{1,2 *}$ \\ ${ }^{1}$ INSERM U862, Magendie Neurocenter, Neurogenesis and Pathophysiology Group, Bordeaux F-33077, France, and ${ }^{2}$ University of Bordeaux, Bordeaux \\ F-33077, France
}

In the mammalian brain, the dentate gyrus and the olfactory bulb are regions where new neurons are continuously added. While the functional consequences of continuous hippocampal neurogenesis have been extensively studied, the role of olfactory adult-born neurons remains elusive. In particular, the involvement of these newborn neurons in odor processing is still a matter of debate. We demonstrate a critical impact of both the age of new neurons and the memory processes involved (learning vs recall) in the recruitment of newborn cells. Thus, odor stimulation preferentially recruited immature neurons over more mature ones ( 2 weeks old vs 5 and 9 weeks old), whereas associative learning based on odor discrimination preferentially recruited mature neurons (5-9 weeks old). Furthermore, while mature neurons were activated by this associative learning, they were not activated by long-term memory recall, indicating that the contribution of newborn neurons in olfactory functions depends also on the memory process involved. Our data thus show that newborn neurons are indeed involved in odor processing and that their recruitment is age- and memory process-dependent.

\section{Introduction}

Along with the dentate gyrus (DG) of the hippocampal formation, the olfactory bulb $(\mathrm{OB})$ is a region of the mammalian brain in which there is a continued supply of newly generated neurons (Abrous et al., 2005; Lledo et al., 2008). Olfactory adult-born neurons originate from the periventricular region, and then migrate tangentially through the rostral migratory stream to reach the $\mathrm{OB}$. Within this structure, they migrate radially into the granular and periglomerular layers, in which they differentiate into GABAergic and dopaminergic neurons (Luskin, 1993; Lois and Alvarez-Buylla, 1994; Alvarez-Buylla and GarciaVerdugo, 2002). During this step, nearly half of the interneurons die within 15-45 d after their birth (Petreanu and Alvarez-Buylla, 2002; Winner et al., 2002; Yamaguchi and Mori, 2005). After this period of death, the surviving cells are integrated into the OB network (Petreanu and Alvarez-Buylla, 2002; Belluzzi et al., 2003; Carleton et al., 2003), suggesting that they may sustain olfactory functions.

Most studies addressing the functional role of olfactory adultborn neurons have highlighted a participation of granular cells. Thus, the first studies, which relied on correlative approaches, have shown that conditions increasing the number of newborn granular cells improve olfactory memory (Rochefort et al., 2002; Rochefort and Lledo, 2005; Moreno et al., 2009; Veyrac et al.,

Received July 29, 2010; revised Nov. 5, 2010; accepted Nov. 6, 2010.

This work was supported by the French National Institute of Health and Medical Research (INSERM), and the University of Bordeaux 2. L.B. was a recipient of a Fondation pour la Recherche Médicale Aquitaine fellowship. We greatly acknowledge C. Dupuy and S. Rey for their technical help. The microscopy was done at the Bordeaux Imaging Center. The help of C. Poujol, S. Marais, P. Legros, and L. Malicieux is acknowledged.

*D.N.A. and M.K. contributed equally to this work.

Correspondence should be addressed to Muriel Koehl, INSERM U862, Magendie Neurocenter, Neurogenesis and Pathophysiology Group, 146 rue Léo Saignat, Bordeaux F-33077, France. E-mail: muriel.koeh@@inserm.fr.

DOI:10.1523/JNEUROSCI.3941-10.2011

Copyright $\odot 2011$ the authors $\quad 0270-6474 / 11 / 311010-07 \$ 15.00 / 0$
2009), while conditions reducing it decrease olfactory performances (Gheusi et al., 2000; Enwere et al., 2004; Bath et al., 2008). More recently, causal approaches relying on depletion of bulbar adult-born neurons by irradiation, anti-mitotic treatment, or genetic tools led to contradictory results. Indeed, irradiated mice present long-term memory alterations (Lazarini et al., 2009), antimitotic-treated mice exhibit short-term memory deficits (Breton-Provencher et al., 2009), and finally olfactory functions are spared in transgenic Nes-CreER ${ }^{\mathrm{T} 2}$ /NSE-DTA mice, in which all the newborn cells die as soon as they differentiate in neuroblast (Imayoshi et al., 2008). Thus, the exact function of newborn granular neurons remains unclear.

Here we hypothesized that the functional implication of adult-born granular neurons may depend on their age and/or the memory process required to perform a given behavioral task. We first analyzed how granular neurons of different ages $(2,5$, or 9 weeks) contribute to an associative learning based on odor discrimination. This odor discrimination task was shown to specifically involve granule cells at the level of the olfactory bulb (Abraham et al., 2004, 2010). We used a functional anatomy approach and mapped the activation of newborn cells with the immediate early gene (IEG) cfos, a marker of cellular activation that has been previously used to image activation within the olfactory bulb (Guthrie et al., 1993; Sallaz and Jourdan, 1996; Roullet et al., 2004), and in particular activation of adult-born granular neurons (Magavi et al., 2005). Then after showing that 5 -week-old neurons were preferentially recruited by this associative learning, we examined whether this preferential recruitment favors the subsequent contribution of these neurons to long-term odor memory recall 1 month later.

We found that while a simple olfactory stimulation preferentially recruits the younger neurons (2 weeks), associative learning based on odor discrimination preferentially recruits older 5- to 
9-week-old neurons, with a peak of recruitment for 5-week-old ones. However, these latter neurons were not recruited 4 weeks later by long-term odor memory recall. Our results thus highlight that newborn neurons are involved in odor processing and that their involvement is age- and process-dependent.

\section{Materials and Methods}

Animals

Three-month-old male C57BL/6J mice (Charles River) were individually housed under a $12 \mathrm{~h}$ light/ $12 \mathrm{~h}$ dark cycle (lights on from 8:00 A.M. to 8:00 P.M.) in a temperature- $\left(22 \pm 3^{\circ} \mathrm{C}\right)$ and humidity-controlled facility. Animals had ad libitum access to food and water for at least 2 weeks after arrival, after which they were subjected to water restriction (1.5 $\mathrm{ml} / \mathrm{d}$ ) until behavioral experiments. All experimental designs and procedures are in agreement with the guidelines of the animal ethics committee of the Ministère de l'Agriculture (France). Studies were conducted in the Magendie Neurocenter (Bordeaux, France), in strict compliance with the European Community's council directive of 24 November 1986 (86/ 609/ECC) and institutional regulations.

\section{BrdU administration}

Mice were injected with bromodeoxyuridine (BrdU, Sigma), a marker of DNA synthesis ( $50 \mathrm{mg} / \mathrm{kg}$, i.p., in $9 \mathrm{~g} / \mathrm{L}$ saline solution). One daily injection was administered, on 2 consecutive days, 2, 5, or 9 weeks before behavioral training (Fig. 1A). This procedure was chosen to precisely label a population of newborn neurons that will be 2,5 , or 9 weeks old at the time of training.

\section{Odor discrimination learning}

Using an olfactometer (Knosys), standard operant procedures (Bodyak and Slotnick, 1999) were used to train mice in a go/no-go discrimination task (Fig. $1 B$ ). Briefly, during the first $3 \mathrm{~d}$, mice were trained to lick a water-reinforced tube ( $5 \mu \mathrm{l}$ of water delivered per lick) when odor $\mathrm{S}^{+}$ ( $1 \%$ isoamylacetate in mineral oil, $50 \mathrm{CC} / \mathrm{min}$ ) was presented; during this training phase, animals underwent on average 90 trials per day; on the fourth day, i.e., the test day, the $\mathrm{S}^{-}$stimulus ( $1 \%$ eucalyptus in mineral oil, $50 \mathrm{CC} / \mathrm{min}$ ) was introduced, and animals learned to refrain from licking when $\mathrm{S}^{-}$was presented. During this discrimination phase, trials $\mathrm{S}^{+}$and $\mathrm{S}^{-}$were presented in a random order, and testing was stopped when the animals performed two successive blocks (each block consists of $10 \mathrm{~S}^{+}$and $10 \mathrm{~S}^{-}$trials) of at least $85 \%$ of success (paired group: $\mathrm{P}$ ). To control for the effects of olfactory stimulation and training, an unpaired group $(\mathrm{U})$ was created, for which $\mathrm{S}^{+}$and $\mathrm{S}^{-}$odors were reinforced randomly. Unpaired mice were matched with mice from the paired group to receive the same amount of trials. These mice thus received the same odor stimulation than mice from the paired group from which they differ in that they were not required to discriminate the two odors to receive a water reward, which was randomly distributed between trials with $\mathrm{S}^{+}$and trials with $\mathrm{S}^{-}$. As a consequence, unpaired mice lick almost constantly the water tube whatever the odor presented. Thus, their percentage of correct responses is 100 for odor $\mathrm{S}^{+}$, and 0 for odor $\mathrm{S}^{-}$, with a mean of $50 \%$, i.e., random performance. To determine basal Fos levels, an additional control group (C) of unmanipulated animals was constituted. All groups were water restricted $(1.5 \mathrm{ml} / \mathrm{d})$ for at least $10 \mathrm{~d}$ before behavioral training. Once training started, unpaired and paired mice received water only through the reinforcements received in the olfactometer, and control mice were fed the same amount of water to control for the effects of water deprivation.

\section{Long-term memory test}

One month after discrimination learning, a batch of mice, for which olfactory learning took place 5 weeks after BrdU injection, was tested for one block in the olfactometer (see Fig. $4 A$ ). $\mathrm{S}^{+}$and $\mathrm{S}^{-}$trials were presented in a pseudorandomized order, and odor $\mathrm{S}^{+}$was never rewarded. Four groups were made. The first was a paired-recall group, in which the animals that had acquired the olfactory discrimination learning were exposed to $\mathrm{S}^{+}$and $\mathrm{S}^{-}$odors 1 month later (P-Recall). To control for the impact of olfactory stimulation, a paired-no-recall group was constituted, in which the animals that had acquired olfactory learning were placed 1 month later in the olfactometer but not exposed to odors (P-No Recall). To control for the impact of initial discrimination learning, an unpaired-recall group was constituted, in which animals had not acquired the olfactory discrimination learning but were tested in the olfactometer (U-Recall). The fourth and last group was a control unmanipulated group $(\mathrm{C})$.

\section{Immunohistochemistry}

Animals were killed $1 \mathrm{~h}$ after the test exposure, on the fourth day, and immunohistochemistry was performed on all animals used in the behavioral experiments. They were anesthetized with pentobarbital $(100 \mathrm{mg} /$ $\mathrm{kg}$ ) and perfused transcardially with $30 \mathrm{ml}$ of PBS, $\mathrm{pH} 7.3$, before $30 \mathrm{ml}$ of $4 \%$ paraformaldehyde in PBS, $\mathrm{pH}$ 7.3. Following a $5 \mathrm{~d}$ postfixation period in paraformaldehyde, $40 \mu \mathrm{m}$ sagittal sections were cut on a vibratome and collected in PBS-azide (0.02\%).

For BrdU and Fos immunohistochemistries, sections were processed according to a standard procedure (Koehl et al., 2008). Briefly, for each staining, one in 10 free-floating sections were incubated with a rat monoclonal anti-BrdU (1/1000, Accurate) or a rabbit polyclonal anti-Fos antibody (1/4000, Calbiochem). Then, sections were incubated with a biotin-labeled goat anti-rat (1/1000, GE Healthcare) or biotin-labeled goat anti-rabbit (1/500, Dako) secondary antibodies. Immunoreactivities were visualized by the biotin-streptavidin technique (ABC kit; Dako) with 3,3'-diaminobenzidine and 3,3'-diaminobenzidine-nickel chloride, respectively for BrdU and Fos, as chromogen.

For double or triple labeling, sections were treated with $2 \mathrm{~N} \mathrm{HCl}$ (30 $\min$ at $37^{\circ} \mathrm{C}$ ) and incubated with a mixture of rat monoclonal anti-BrdU antibody (1/1000; Accurate), mouse monoclonal anti-NeuN antibody (1/1000, Millipore Bioscience Research Reagents), and rabbit polyclonal anti-Fos antibody (1/5000, Calbiochem). For double labeling, only anti$\mathrm{BrdU}$ and anti-Fos antibodies were used. Immunoreactivities were revealed with Cy3 goat anti-rat (1/1000, Jackson), Cy5 goat anti-mouse (1/1000, Molecular Probe), and Alexa 488 goat anti-rabbit (1/1000, Molecular Probe) secondary antibodies.

\section{Stereological analysis of BrdU staining}

The number of BrdU-IR cells was quantified in the left hemisphere under $1000 \times$ magnification with the optical fractionator method on a systematic random sampling of every tenth section along the temporomedian axis. We verified in a preliminary experiment that sampling of every fifth section (200 $\mu \mathrm{m}$ apart, $\sim 7$ sections of OB) and every tenth section (400 $\mu \mathrm{m}$ apart, $\sim 4$ sections of $\mathrm{OB})$ yielded the same results $(33,300 \pm 2230.94$ cells and $34,848 \pm 2750.38$ cells for the $200 \mu \mathrm{m}$ and the $400 \mu \mathrm{m}$ condition, respectively, $n=15$ mice; $t_{(14)}=1.71, p=0.12$ ). For all the age conditions, BrdU-IR cells were counted in $40 \times 40 \mu \mathrm{m}$ counting frames at evenly spaced intervals of $200 \times 200 \mu \mathrm{m}$, with an exclusion guard zone of $2 \mu \mathrm{m}$ for both counting frames (Stereo Investigator software, MicroBrightField).

\section{Quantification of double/triple-labeled cells}

All analyses were performed with a confocal microscope (Leica DMR TCS SP2 AOBS) equipped with a $40 \times$ oil-immersion objective, an argon laser $(488 \mathrm{~nm})$, a green helium-neon laser $(543 \mathrm{~nm})$, and a red heliumneon laser $(633 \mathrm{~nm})$. For the $\mathrm{OB}$, confocal acquisitions were performed with a $1.7 \times$ zoom along the entire $z$-axis $(25 \mu \mathrm{m})$ using $1 \mu \mathrm{m}$ intervals, and were analyzed with the MetaMorph software (Roper Scientific). Briefly, background was subtracted, a median filter was applied to gloss the images, and for each staining, images were overlaid, plane by plane, to determine colocalization of markers. For each animal, the number of BrdU-IR cells analyzed corresponded to $25 \%$ of the total BrdU-IR cells, as estimated with DAB staining, thus ranging from 200 to 500 cells.

For discrimination learning, the percentages of activated newborn neurons in the granular cell layer (GCL) were determined by triple BrdUNeuN-Fos immunostaining. For long-term memory recall, the activation of newborn neurons was determined by a double BrdU-Fos immunofluorescent staining.

\section{Quantification of Fos-IR cells}

Fos expression was quantified by measuring optical density (OD) with ImageJ software (http://rsbweb.nih.gov/ij/) for 1 in every 10 sagittal sec- 


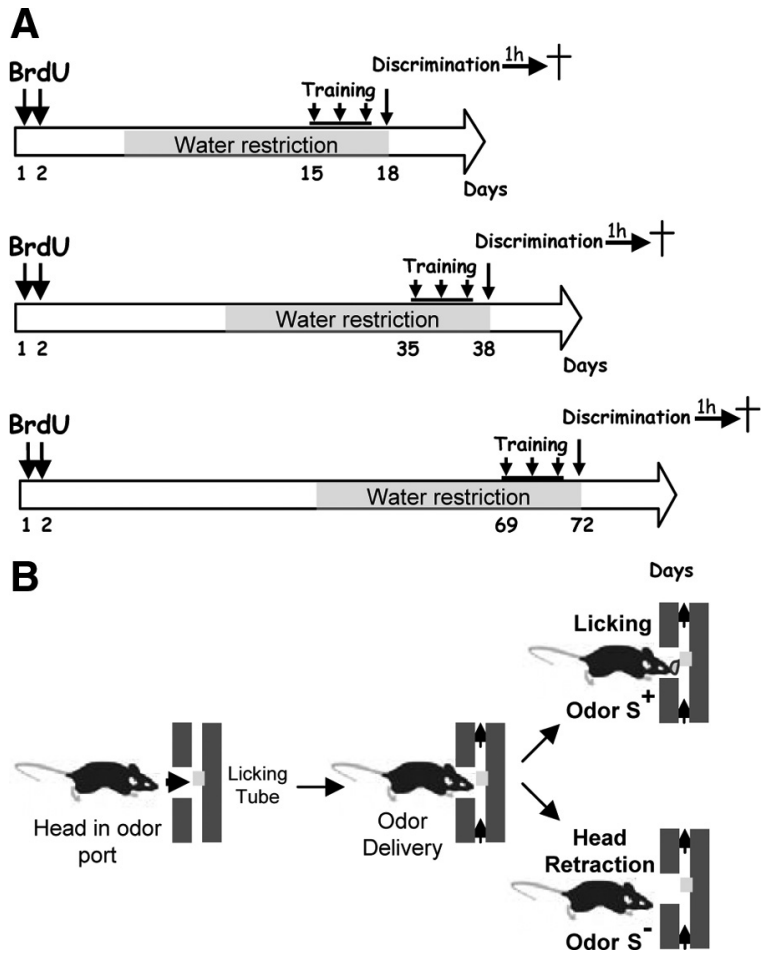

Figure 1. Scheme of the experiment. $A$, Experimental design of the olfactory discrimination learning task. $\boldsymbol{B}$, Schematic representation of the go/no-go task.

tions. For each OB, the granular zone to be analyzed was delimited, and the density of staining was calculated by dividing the pixel count by the granular zone area (pixels per $\mu \mathrm{m}^{2}$ ).

\section{General procedures}

Experiment 1: time course of newborn neuron recruitment by olfactory discrimination learning. Mice were injected with BrdU 2, 5, or 9 weeks before olfactory learning. In each condition they were subjected to water restriction, starting $12 \mathrm{~d}$ before learning (Fig. 1A). Olfactory learning lasted $4 \mathrm{~d}$, including $3 \mathrm{~d}$ of training and $1 \mathrm{~d}$ of discrimination learning performed when the newborn BrdU-IR cells were 2, 5, or 9 weeks old. At the end of the test, animals were placed back in the animal room, and killed $1 \mathrm{~h}$ later.

Experiment 2: recruitment of olfactory newborn neurons by long-term memory recall. Mice underwent the same procedure as before (5-weekold condition), and were retested in the olfactometer 1 month later (day 69) (see Fig. 4A). At the end of the test, animals were placed back in the animal room, and killed $1 \mathrm{~h}$ later.

\section{Statistical analyses}

All statistical analyses were performed with Statistica 8.0 software (Statsoft). Olfactometer and immunohistochemistry data were analyzed with two-way or one-way ANOVA and Student's $t$ test. Whenever appropriate, ANOVAs were followed by post hoc comparisons using the Newman-Keuls test. Data in the bar graphs and in the text are presented as mean \pm SEM.

\section{Results}

\section{Olfactory discrimination learning preferentially recruits} mature, 5- to 9-week-old adult-born neurons

Here we examined whether immature ( 2 weeks old) or mature ( 5 or 9 weeks old) adult-born bulbar granular neurons are recruited during olfactory discrimination learning.

To do so, animals were injected with BrdU 2, 5, or 9 weeks before testing (Fig. 1A). Then for $3 \mathrm{~d}$ they were trained to associate an odor with water reinforcement; on the fourth day, a new odor was introduced for which animals have to learn that it is not reinforced in a go/no-go procedure (Fig. $1 B$ ). To control for the effects of olfactory stimulation and training, an unpaired group (U) was created, for which the two odors were reinforced randomly on the testing day.

Behavioral results showed that during learning, $\mathrm{P}$ mice from the three age conditions $(n=6,7$, and 8 mice for the 2,5 , and 9 week conditions, respectively) readily improved their performances in the discrimination task (Fig. 2A) (group effect, $F_{(2,18)}=3.14, p=0.07$; block effect, $F_{(4,72)}=72.99, p<0.001$; group $\times$ block $\left.F_{(8,72)}=0.35, p=0.94\right)$ and reached a stable level of $85 \%$ success in $4-5$ blocks of trials.

We next investigated the effect of discrimination learning on the survival and differentiation of granular newborn cells. We found that the number of BrdU-IR cells was age dependent (age effect, $\left.F_{(2,60)}=45.26, p<0.05\right)$. Indeed, the number of BrdU-IR cells in the 5 and 9 week conditions was lower than in the 2 week condition ( 2 weeks vs 5 weeks, $p<0.001$ and 2 weeks vs 9 weeks, $p<0.001 ; 5$ weeks vs 9 weeks, $p=0.46)$. However, it did not differ between groups (Fig. $2 B, C$ ) (group effect, $F_{(2,60)}=0.17$, $p=0.84$, group $\times$ age, $F_{(4,60)}=1.44, p=0.23$; mean $\pm \mathrm{SEM}$ number of BrdU-IR cells: at 2 weeks, $C=50,334 \pm 4207, n=9$; $\mathrm{U}=56,464 \pm 5006, n=7 ; p=47,062 \pm 3387, n=6$; at 5 weeks, $\mathrm{C}=27,825 \pm 2925, n=6 ; \mathrm{U}=27,891 \pm 1774, n=8 ; p=$ $26,196 \pm 2695, n=7$; at 9 weeks, $\mathrm{C}=28,916 \pm 2563, n=9 ; \mathrm{U}=$ $26,417 \pm 2862, n=9 ; p=33,000 \pm 3312, n=8)$. The analysis of cell differentiation indicated that whatever the age condition and the experimental group, the percentage of BrdU-IR cells expressing the neuronal marker NeuN was similar ( 95\%) (Fig. 2D) (group effect, $F_{(2,52)}=1.59, p=0.21$; age effect, $F_{(2,52)}=1.25$, $p=0.30$; group $\times$ age, $F_{(4,52)}=1.21, p=0.32$; mean \pm SEM percentage of BrdU-NeuN-IR cells: at 2 weeks, $C=95.31 \pm 0.27$, $n=9 ; \mathrm{U}=94.19 \pm 0.40, n=7 ; p=94.91 \pm 0.35, n=6$; at 5 weeks, $\mathrm{C}=92.74 \pm 1.09, n=6 ; \mathrm{U}=93.66 \pm 1.74, n=8 ; p=$ $96.05 \pm 0.92, n=7$; at 9 weeks, $\mathrm{C}=94.77 \pm 0.81, n=6$; $\mathrm{U}=$ $95.58 \pm 0.75, n=7 ; p=95.81 \pm 0.53, n=5)$.

To determine the effect of olfactory discrimination learning on the activation of 2-, 5-, and 9-week-old granular neurons, we analyzed the percentage of newborn neurons (those expressing both BrdU and NeuN) that also expressed Fos (Fig. $3 A, B$ ). Whatever the age of the newborn cells, the percentage of activated neurons was elevated in both groups exposed to the test odors ( $\mathrm{U}$ and $\mathrm{P}$ ) compared to $\mathrm{C}$ animals (group effect; $F_{(2,52)}=59.70, p<$ 0.0001; C vs U, $p<0.001$; C vs P, $p<0.001$; mean \pm SEM percentage of BrdU-NeuN-Fos-IR cells: at 2 weeks, $\mathrm{C}=1.28 \pm$ $0.52 \%$ of 350 cells analyzed, $n=9 ; \mathrm{U}=14.88 \pm 1.35 \%$ of 370 cells analyzed, $n=7 ; p=12.91 \pm 1.00 \%$ of 375 cells analyzed, $n=6$; at 5 weeks, $\mathrm{C}=1.13 \pm 0.09 \%$ of 250 cells analyzed, $n=6$; $\mathrm{U}=$ $7.59 \pm 1.37 \%$ of 345 cells analyzed, $n=8 ; p=14.05 \pm 2.01 \%$ of 360 cells analyzed, $n=7$; at 9 weeks, $\mathrm{C}=0.76 \pm 0.37 \%$ of 210 cells analyzed, $n=6 ; \mathrm{U}=3.10 \pm 0.47 \%$ of 250 cells analyzed, $n=$ $7 ; p=5.33 \pm 1.12 \%$ of 300 cells analyzed, $n=5$ ). In particular, the activation of newborn neurons by olfactory stimulation (unpaired mice) differed as a function of neuron's age (age effect; $\left.F_{(2,52)}=25.80, p<0.0001\right)$. Indeed the percentage of BrdUlabeled neurons expressing Fos, which reached $\sim 14 \%$ for 2 -week-old cells (U 2 weeks $=14.88 \pm 1.35 \%, n=7$ ), was halved in 5 -week-old ones (U 5 weeks $=7.59 \pm 1.37 \%, n=8$ ) and halved again in 9-week-old ones (U 9 weeks $=3.10 \pm 0.47 \%, n=$ 7; U 2 weeks vs U 5 weeks, $p<0.001$; U 5 weeks vs U 9 weeks, $p<$ $0.05)$. These results indicate that simple olfactory stimulation preferentially recruited the younger neurons.

More importantly, the recruitment of newborn neurons by discrimination learning (comparison $\mathrm{P}$ vs $\mathrm{U}$ ) differed as a 
A

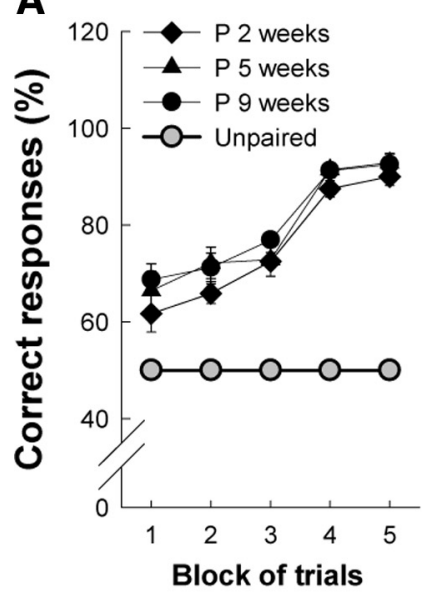

C

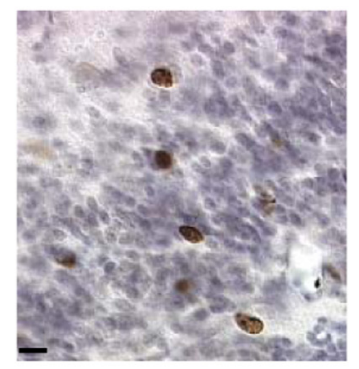

B

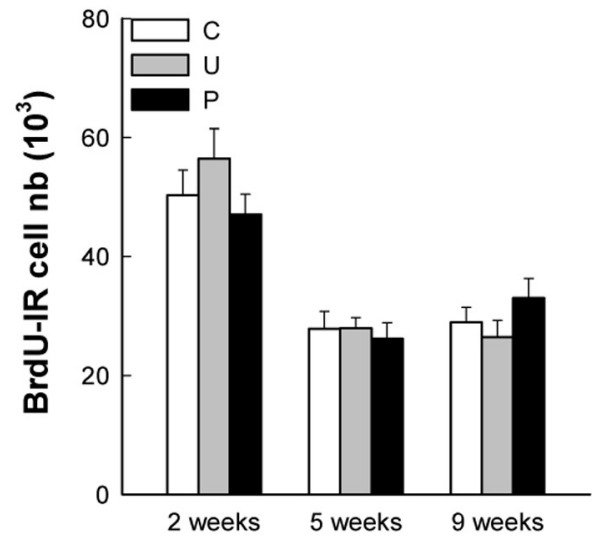

D

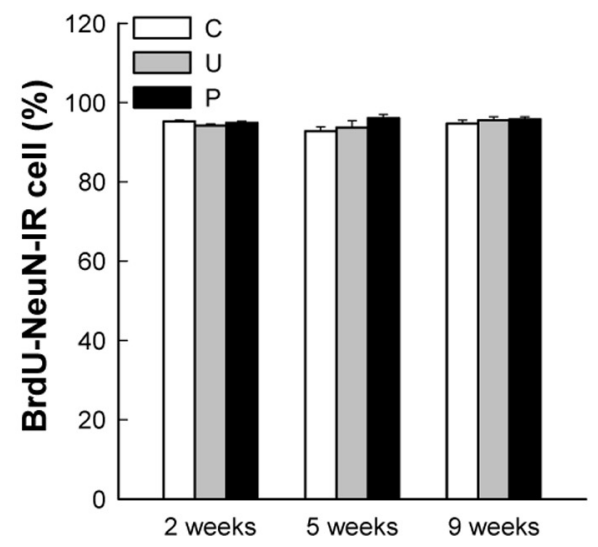

Figure 2. Olfactory discrimination learning has no impact on survival and differentiation of 2-, 5-, and 9-week-old olfactory granular neurons. $\boldsymbol{A}$, Behavioral performances in the olfactory discrimination learning task. $\boldsymbol{B}$, Number of BrdU-IR cells. $\boldsymbol{C}$, Illustration of BrdU-IR cells in the GCL of counterstained bulbar sagittal sections. $D$, Percentage of BrdU-IR cells expressing the neuronal marker NeuN in the GCL. $n=6-9$ per group. Scale bar, $10 \mu \mathrm{m}$.

ing granule neurons expressing Fos was much lower than that of newborn ones (neuronal population effect; $F_{(1,18)}=$ 49.24, $p<0.0001$; mean \pm SEM percentage of NeuN-Fos-IR cells: $\mathrm{C}=$ $0.73 \pm 0.44$ and $1.13 \pm 0.09$ for preexisting and newborn neurons, respectively, $n=6 ; \mathrm{U}=1.61 \pm 0.35$ and $7.59 \pm 1.37$ for preexisting and newborn neurons, respectively, $n=8 ; p=2.92 \pm$ 0.63 and $14.05 \pm 2.01$ for preexisting and newborn neurons, respectively, $n=$ 7), and that the impact of training/ learning was not the same in preexisting versus newborn neurons (group $\times$ neuronal population effect; $F_{(2,18)}=12.95$, $p<0.0001)$. In particular, whereas unpaired and paired mice did not differ in the percentage of preexisting neurons activated (NK post hoc test, $p=0.40$; $\mathrm{U}=1.61 \pm 0.35, n=8 ; p=2.92 \pm 0.63$, $n=7$ ), they did differ in the percentage of newborn neurons activated (NK post hoc test, $p<0.001 ; \mathrm{U}=7.59 \pm 1.37, n=$ $8 ; p=14.05 \pm 2.01, n=7)$, indicating that adult-born olfactory granule neurons have a greater Fos response to discrimination learning than mature preexisting neurons.

Altogether, these data indicate that there is a critical time window for bulbar newborn neuron recruitment by olfactory processing: olfactory stimulation recruits immature neurons (2 weeks), whereas discrimination learning preferentially recruits more mature ones (5- and 9-weekold neurons). function of neuron's age (group $\times$ age; $F_{(4,52)}=9.55, p<$ $0.0001)$. Whereas discrimination learning did not recruit 2-week-old neurons $\left(F_{(2,19)}=65.46, p<0.001, \mathrm{P}\right.$ vs $\mathrm{U}, p=0.17 ; \mathrm{U}=14.88 \pm$ $1.35 \%, n=7 ; p=12.91 \pm 1.00 \%, n=6$ ), it activated 5-week-old $\left(F_{(2,18)}=17.68, p<0.001, \mathrm{P}\right.$ vs $\mathrm{U}, p<0.001 ; \mathrm{U}=7.59 \pm 1.37 \%, n=$ $8 ; p=14.05 \pm 2.01 \%, n=7$ ) and 9-week-old ones (group effect: $F_{(2,15)}=11.16, p<0.001, \mathrm{P}$ vs $\mathrm{U} p<0.05 ; \mathrm{U}=3.10 \pm 0.47 \%, n=$ $7 ; p=5.33 \pm 1.12 \%, n=5$ ), with a more pronounced effect in 5 -week-old neurons. To ensure that these differences in percentage of activated neurons after training were not due to differences in the overall activation of the olfactory bulb, we evaluated the expression level of Fos in the granular cell layer (Fig. 3C). We found that training increased Fos level to the same extent in $\mathrm{U}$ and $\mathrm{P}$ groups (group effect; $F_{(2,48)}=45.24, p<0.0001$ with $\mathrm{C}<$ $\mathrm{P}=\mathrm{U}$; age effect; $F_{(2,48)}=0.93, p=0.40$; group $\times$ age; $F_{(4,48)}=$ $1.34, p=0.27$; mean \pm SEM Fos level: at 2 weeks, $\mathrm{C}=0.97 \pm$ $0.29, n=9 ; \mathrm{U}=3.69 \pm 0.46, n=7 ; p=2.83 \pm 0.23, n=6$; at 5 weeks, $\mathrm{C}=0.76 \pm 0.10, n=6 ; \mathrm{U}=2.67 \pm 0.28, n=7 ; p=3.42 \pm$ $0.66, n=5$; at 9 weeks, $\mathrm{C}=0.61 \pm 0.30, n=6 ; \mathrm{U}=2.87 \pm 0.31$, $n=6 ; p=2.88 \pm 0.23, n=5$ ), suggesting that the difference in activation between paired and unpaired mice was specific to newborn cells. To analyze this specificity, we next compared the activation of preexisting neurons $\left(\mathrm{BrdU}^{-} \mathrm{NeuN}^{+}\right.$cells also expressing Fos) to that of newborn neurons in the 5-week-old condition (Fig. 3D). We found that the percentage of preexist-
Long-term memory recall does not recruit 9-week-old newborn neurons exposed to learning at $\mathbf{5}$ weeks of age We next tested whether newborn neuron recruitment depends on the memory process involved in the task (i.e., odor discrimination learning vs odor memory recall). To this end, mice were trained in the discrimination learning paradigm 5 weeks after BrdU injection, a time at which their recruitment was more pronounced, and tested for long-term memory recall 1 month later (Fig. 4A).

During the learning phase, mice from the P groups $(n=6$ mice for both the P-No Recall and the P-Recall groups) rapidly improved their behavioral performances (Fig. $4 B$ ) (block effect; $F_{(4,40)}=44.08, p<0.0001$ ) and did not differ at this stage (group effect, $F_{(1,10)}=1.66, p=0.23$; group $\times$ block, $F_{(4,40)}=1.46, p=$ $0.23)$. One month later, when mice were retested in the olfactometer, animals from the P-Recall group displayed a 74\% correct response rate and differed from mice of the U-Recall group, which remained at chance level (Fig. 4C) (mean \pm SEM percentage of correct responses: U-Recall $=55.00 \pm 6.80, n=6$; $\mathrm{P}$-Recall $\left.=74.17 \pm 3.16, n=6 ; t_{(9)}=2.40, p<0.05\right)$.

As expected, the number of BrdU-IR cells was similar in the four groups (Fig. $4 D)\left(F_{(3,18)}=0.19, p=0.90\right.$; mean \pm SEM BrdU-IR cell number: $\mathrm{C}=30,749 \pm 4100, n=5$; U-Recall $=$ $27,525 \pm 4937, n=5$; P-No Recall $=27,250 \pm 4145, n=6$; P-Recall $=29,688 \pm 2155, n=6)$. Whatever the experimental 
treatment (i.e., whether animals had previously learned to discriminate odors or not, and whether they were exposed or not to odors during the memory test), newborn cell activation remained very low $(<2 \%)$, and did not differ between groups (Fig. $4 E)\left(F_{(3,18)}=0.74, p=0.54\right.$; mean \pm SEM percentage of BrdU-NeuNFos-IR cells: $\mathrm{C}=0.38 \pm 0.15 \%$ of 430 cells analyzed, $n=5$; U-Recall $=1.21 \pm 0.66 \%$ of 388 cells analyzed, $n=5$; P-No Recall $=$ $0.69 \pm 0.39 \%$ of 490 cells analyzed, $n=6$; P-Recall $=0.66 \pm 0.20 \%$ of 400 cells analyzed, $n=6)$.

\section{Discussion}

Although considerable information is available concerning the functional role of adult-born granular neurons of the olfactory bulb, no consensus arises regarding their implication in odor processing. Using a functional imaging approach, we show here that the contribution of olfactory newborn granular neurons in olfactory function depends on their age and on the memory process: olfactory stimulation preferentially recruits immature over more mature newborn neurons (2 weeks old vs 5 and 9 weeks old), whereas associative learning based on odor discrimination preferentially recruits older ones ( 5 and 9 weeks old). In addition, while mature neurons are activated by discrimination learning, they are not reactivated by long-term memory recall.

\section{Impact of odor processing on bulbar neurogenesis}

During their integration into the bulbar network, a vast majority of newborn cells are submitted to cell death. As expected from studies placing the death period of granular cells between 15 and 45 d of age, i.e., 2 and 7 weeks (Petreanu and Alvarez-Buylla, 2002; Winner et al., 2002; Yamaguchi and Mori, 2005), we report here a decrease in the number of cells between 2 and 5 weeks of age. Whatever the age of cells, this rate of cell survival was affected neither by odor stimulation nor by odor discrimination learning, a result inconsistent with some previous reports (Mandairon et al., 2006; Mouret et al., 2008). Indeed, from the Mouret et al. (2008) and Mandairon et al. (2006) studies, an increase in neurogenesis was expected for 2-week-old cells, a decrease for 5-week-old ones, and no differences for the 9-week-old cells. However, Mouret et al. (2008) reported that the olfactometer training procedures, where mice learn to lick the reward tube when mineral oil is delivered, did not per se alter neuronal survival. When comparing with our procedure, both paired and unpaired mice undergo this training for $3 \mathrm{~d}$, and the absence of cell survival effect is thus consistent with the results of Mouret et al. (2008). Finally, although our training procedure involved an odor and not mineral oil, the length of training and the number of trials per day is much lower in our study (3 $\mathrm{d}$ with a mean of 90 trials /d) than in other studies [7 d, 200 trials/d (Mouret et al., 2008)]. Altogether, these data indicate that newborn cell survival depends not only on the age of cells but also on the degree of sensory input. As cell survival, neuronal differentiation was not influenced by learning in our

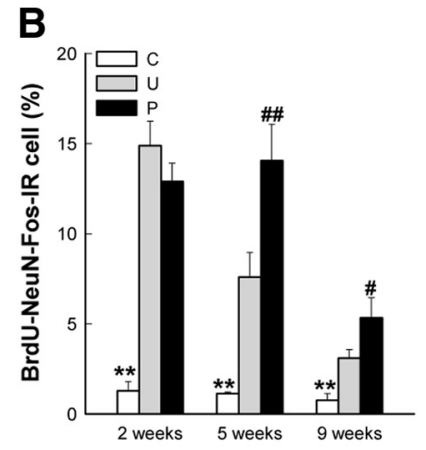

D
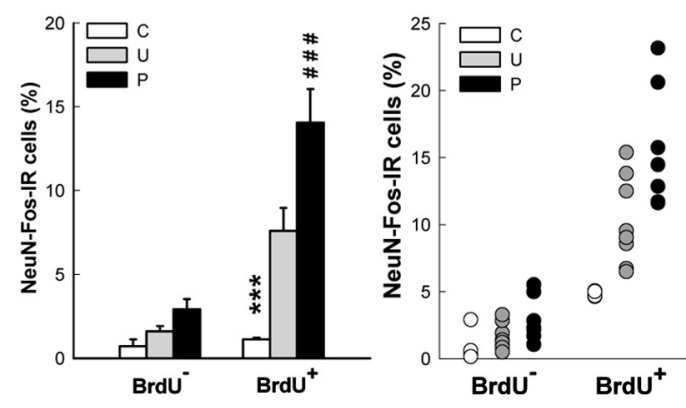

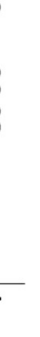

Figure 3. Olfactory discrimination learning preferentially recruits 5 -week-old neurons in the $0 B$. $A$, Confocal illustration showmeans and individual data are presented. ${ }^{* *} p<0.01$ C versus $U$ and $P,{ }^{* * *} p<0.001$ C versus $U$ and $P ;{ }^{*} p<0.05 U$ versus $P$
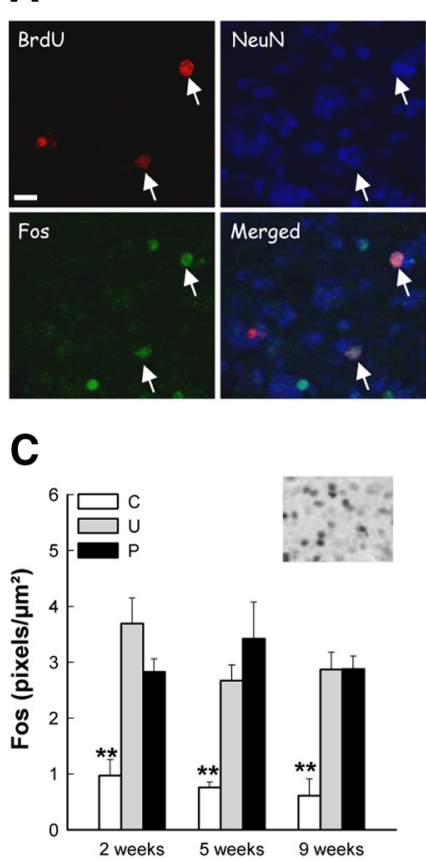

study, which is in agreement with the literature (Mouret et al., 2008; Moreno et al., 2009; Veyrac et al., 2009).

Recruitment of newborn neurons by odor stimulation

By imaging the activation of 2-, 5-, and 9-week-old neurons using a classical functional anatomical approach (Jessberger and Kempermann, 2003; Ramirez-Amaya et al., 2006; Kee et al., 2007; Tashiro et al., 2007; Trouche et al., 2009), we found that olfactory stimulation activates more 2-week-old than 5- and 9-week-old neurons, suggesting that it preferentially recruits younger, more immature neurons. This is in line with previous observations in different experimental conditions showing that exposure to a mixture of odors activates more 2- to 3-week-old neurons than 4to 7-week-old ones (Magavi et al., 2005). Interestingly, it has been shown that such priming of immature neurons (1-3 weeks old) by a mixture of odors improves subsequent odor discrimination, indicating that immature neurons can retain a trace of previous olfactory experience and that they participate in perceptual learning (Moreno et al., 2009).

\section{Recruitment of newborn neurons by odor discrimination learning}

More importantly, we report that associative learning based on odor discrimination activates specifically mature new neurons (5 and 9 weeks old). Indeed, the percentage of newborn neurons expressing Fos was higher in groups that acquired discrimination learning $(\mathrm{P})$ than in animals only exposed to odors $(\mathrm{U})$. As the procedure developed for discrimination learning assesses shortterm associative memory abilities (Slotnick, 2001), this activation of mature new neurons certainly reflects their contribution to short-term memory. This result is in line with a recent study 
A



B

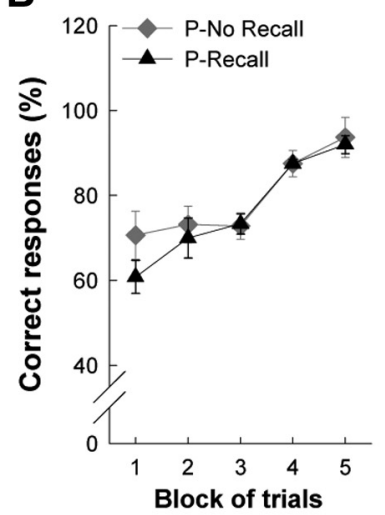

C
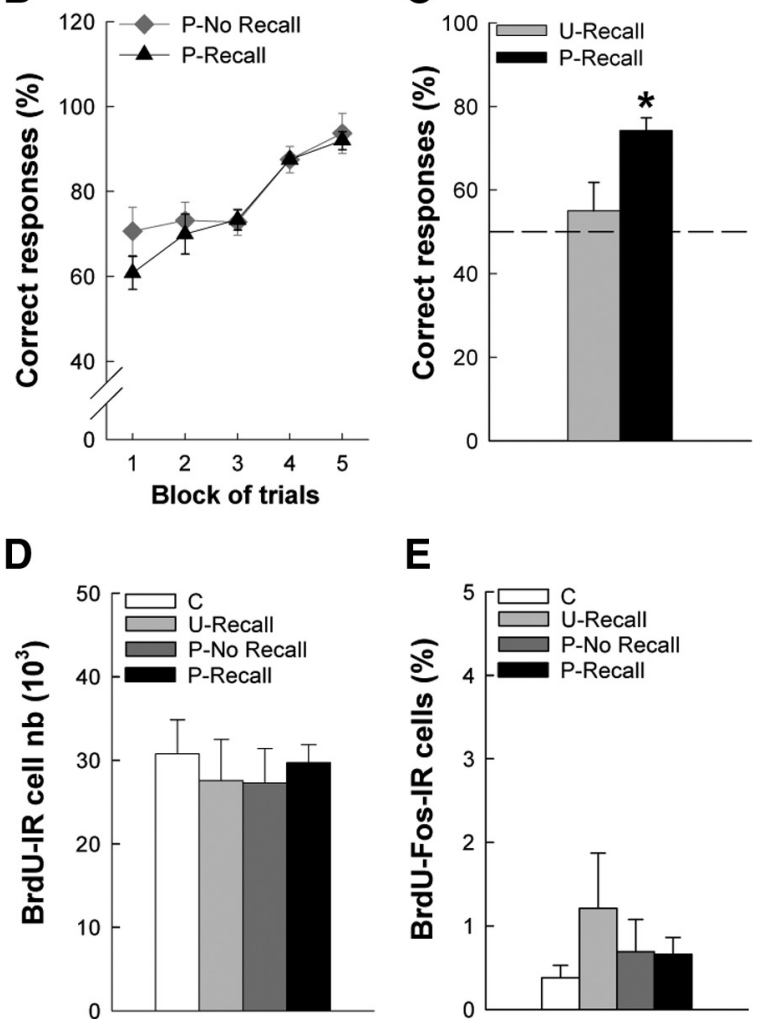

E

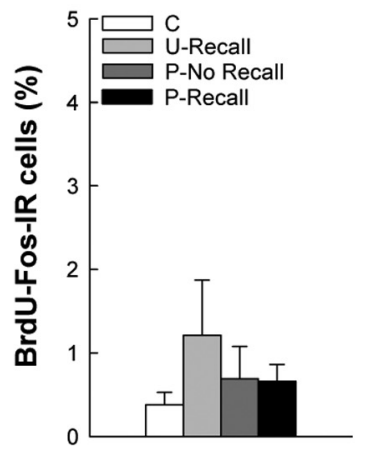

Figure 4. Long-term memory recall does not recruit 9-week-old newborn granular neurons exposed to learning at 5 weeks of age. $A$, Experimental design of the long-term memory recall task. $\boldsymbol{B}, \boldsymbol{C}$, Percentage of correct responses during the discrimination learning phase $(\boldsymbol{B})$ and during memory recall (C). D, Number of BrdU-IR cells. $\boldsymbol{E}$, Percentage of BrdU-Fos-IR cells in the GCL. C, U-Recall, P-No Recall, P-Recall, $n=5-6$ per group; ${ }^{*} p<0.05$.

showing that a 3 week AraC treatment is associated with an alteration of short-term memory (Breton-Provencher et al., 2009). However, in this study, 2 - to 28 -d-old neurons, i.e., both immature and more mature ones, were ablated, and the specific contribution of each population could not be analyzed. Our results thus complete and refine these data, showing that neurons implicated in short-term memory are mature neurons, more precisely 5 -week-old ones. Although we cannot at this stage generalize our results to the discrimination of any pair of odors, studies comparing the effects of different odors or pairs of odors have not reported so far any differences. Thus, the effect of odor stimulation on cell survival and the effect of irradiation were found to be similar whatever the odor tested (Mouret et al., 2008; Lazarini et al., 2009; Moreno et al., 2009). It is thus likely that the same specific recruitment of adult-born granular cells would be observed in response to discrimination learning of pairs of odors other than the one we used.

\section{Recruitment of newborn neurons by long-term odor memory recall}

When animals trained according to the same protocol (BrdU injected 5 weeks before learning) were tested for odor memory

recall, we could not find evidence of a preferential activation of mature neurons ( 9 weeks at the time of testing). Thus, the initial recruitment of 5-week-old cells by discrimination learning does not favor their subsequent recruitment upon long-term memory recall, indicating that mature newborn neurons are not implicated in long-term memory. As these mature 9-week-old neurons are recruited by discrimination learning, this result indicates that newborn cells' contribution to olfactory function is memoryprocess dependent. With respect to the literature on newborn cells' involvement in long-term memory, the lack of activation of mature cells by long-term memory recall that we report seems contradictory with the behavioral performances of SVZ-irradiated mice (Lazarini et al., 2009) that exhibit deficits in longterm memory. However, in this study, the number of immature newborn cells (up to 3 weeks) was decreased, while the number of more mature ( $>5$ weeks old) cells was similar to that of control animals as a result of a compensatory increase in cell survival. Thus, it is possible that the long-term memory impairment observed in irradiated mice is specifically linked to a decrease of immature neurons that have been primed during initial training (see above). On this line, a recent study reported that following an initial discrimination learning, immature neurons are recruited $5 \mathrm{~d}$ later by long-term odor memory recall (Sultan et al., 2010). Thus, together with the literature, our results indicate that immature bulbar newborn neurons, which are sensitive to olfactory stimulation and required for perceptual learning, may be involved in long-term odor memory, while mature ones are crucial for learning. These results are reminiscent to those obtained for hippocampal neurogenesis and spatial learning in the water maze. Indeed, whereas mature neurons appear essential for spatial learning (Kee et al., 2007), immature neurons, which are not involved in learning (Kee et al., 2007), are required for remembering and updating the platform location (Trouche et al., 2009).

\section{Impact of age on newborn cells' functional maturation}

The mechanisms of this age-dependent and learning-relevant recruitment of adult-born granular neurons may be associated with morphological and physiological neuronal maturation. Indeed, during their development, newborn neurons integrate into the olfactory circuits by first developing input synapses that can control their global excitation before developing distal input-output synapses that mediate recurrent inhibition within the bulb (Kelsch et al., 2008). At 2 weeks of age, they receive glutamatergic synaptic inputs from axonal collaterals of the OB's projection neuron while exhibiting only a few GABA output synapses. Thus, in response to an odor, these immature neurons can be activated by OB's projection neurons, but in response to this activation, they cannot exercise a lateral inhibition, which is implicated in olfactory discrimination. Then, from 4 weeks on, they are able to exercise a lateral inhibition (Kelsch et al., 2008), a finding that most probably explains their recruitment during olfactory discrimination processing. Interestingly, 5-week-old neurons are still capable of long-term potentiation, a property that they lose along their maturation (Nissant et al., 2009). The features of 5 -week-old neurons, which can receive messages from OB's projection neurons, exercise lateral inhibition, and are capable of LTP, may explain their unique involvement in odor discrimination learning.

Altogether our results highlight the existence of critical time windows for the recruitment of newborn neurons by odor stimulation, and discrimination learning. They suggest that the unique properties that newborn neurons exhibit in the course of 
their maturation allow them to sustain specific olfactory functions. Future studies targeting specific population of newborn cells in a time-controlled manner are thus needed to decipher the implication of bulbar neurogenesis in olfactory functions.

\section{References}

Abraham NM, Spors H, Carleton A, Margrie TW, Kuner T, Schaefer AT (2004) Maintaining accuracy at the expense of speed: stimulus similarity defines odor discrimination time in mice. Neuron 44:865-876.

Abraham NM, Egger V, Shimshek DR, Renden R, Fukunaga I, Sprengel R, Seeburg PH, Klugmann M, Margrie TW, Schaefer AT, Kuner T (2010) Synaptic inhibition in the olfactory bulb accelerates odor discrimination in mice. Neuron 65:399-411.

Abrous DN, Koehl M, Le Moal M (2005) Adult neurogenesis: from precursors to network and physiology. Physiol Rev 85:523-569.

Alvarez-Buylla A, Garcia-Verdugo JM (2002) Neurogenesis in adult subventricular zone. J Neurosci 22:629-634.

Bath KG, Mandairon N, Jing D, Rajagopal R, Kapoor R, Chen ZY, Khan T, Proenca CC, Kraemer R, Cleland TA, Hempstead BL, Chao MV, Lee FS (2008) Variant brain-derived neurotrophic factor (Val66Met) alters adult olfactory bulb neurogenesis and spontaneous olfactory discrimination. J Neurosci 28:2383-2393.

Belluzzi O, Benedusi M, Ackman J, LoTurco JJ (2003) Electrophysiological differentiation of new neurons in the olfactory bulb. J Neurosci 23:10411-10418.

Bodyak N, Slotnick B (1999) Performance of mice in an automated olfactometer: odor detection, discrimination and odor memory. Chem Senses 24:637-645.

Breton-Provencher V, Lemasson M, Peralta MR 3rd, Saghatelyan A (2009) Interneurons produced in adulthood are required for the normal functioning of the olfactory bulb network and for the execution of selected olfactory behaviors. J Neurosci 29:15245-15257.

Carleton A, Petreanu LT, Lansford R, Alvarez-Buylla A, Lledo PM (2003) Becoming a new neuron in the adult olfactory bulb. Nat Neurosci 6:507-518.

Enwere E, Shingo T, Gregg C, Fujikawa H, Ohta S, Weiss S (2004) Aging results in reduced epidermal growth factor receptor signaling, diminished olfactory neurogenesis, and deficits in fine olfactory discrimination. J Neurosci 24:8354-8365.

Gheusi G, Cremer H, McLean H, Chazal G, Vincent JD, Lledo PM (2000) Importance of newly generated neurons in the adult olfactory bulb for odor discrimination. Proc Natl Acad Sci U S A 97:1823-1828.

Guthrie KM, Anderson AJ, Leon M, Gall C (1993) Odor-induced increases in c-fos mRNA expression reveal an anatomical "unit" for odor processing in olfactory bulb. Proc Natl Acad Sci U S A 90:3329-3333.

Imayoshi I, Sakamoto M, Ohtsuka T, Takao K, Miyakawa T, Yamaguchi M, Mori K, Ikeda T, Itohara S, Kageyama R (2008) Roles of continuous neurogenesis in the structural and functional integrity of the adult forebrain. Nat Neurosci 11:1153-1161.

Jessberger S, Kempermann G (2003) Adult-born hippocampal neurons mature into activity-dependent responsiveness. Eur J Neurosci 18:27072712.

Kee N, Teixeira CM, Wang AH, Frankland PW (2007) Preferential incorporation of adult-generated granule cells into spatial memory networks in the dentate gyrus. Nat Neurosci 10:355-362.

Kelsch W, Lin CW, Lois C (2008) Sequential development of synapses in dendritic domains during adult neurogenesis. Proc Natl Acad Sci U S A 105:16803-16808.

Koehl M, Meerlo P, Gonzales D, Rontal A, Turek FW, Abrous DN (2008) Exercise-induced promotion of hippocampal cell proliferation requires beta-endorphin. FASEB J 22:2253-2262.

Lazarini F, Mouthon MA, Gheusi G, de Chaumont F, Olivo-Marin JC, Lamarque S, Abrous DN, Boussin FD, Lledo PM (2009) Cellular and behavioral effects of cranial irradiation of the subventricular zone in adult mice. PLoS ONE 4:e7017.

Lledo PM, Merkle FT, Alvarez-Buylla A (2008) Origin and function of olfactory bulb interneuron diversity. Trends Neurosci 31:392-400.

Lois C, Alvarez-Buylla A (1994) Long-distance neuronal migration in the adult mammalian brain. Science 264:1145-1148.

Luskin MB (1993) Restricted proliferation and migration of postnatally generated neurons derived from the forebrain subventricular zone. Neuron 11:173-189.

Magavi SS, Mitchell BD, Szentirmai O, Carter BS, Macklis JD (2005) Adultborn and preexisting olfactory granule neurons undergo distinct experience-dependent modifications of their olfactory responses in vivo. J Neurosci 25:10729-10739.

Mandairon N, Sacquet J, Garcia S, Ravel N, Jourdan F, Didier A (2006) Neurogenic correlates of an olfactory discrimination task in the adult olfactory bulb. Eur J Neurosci 24:3578-3588.

Moreno MM, Linster C, Escanilla O, Sacquet J, Didier A, Mandairon N (2009) Olfactory perceptual learning requires adult neurogenesis. Proc Natl Acad Sci U S A 106:17980-17985.

Mouret A, Gheusi G, Gabellec MM, de Chaumont F, Olivo-Marin JC, Lledo PM (2008) Learning and survival of newly generated neurons: when time matters. J Neurosci 28:11511-11516.

Nissant A, Bardy C, Katagiri H, Murray K, Lledo PM (2009) Adult neurogenesis promotes synaptic plasticity in the olfactory bulb. Nat Neurosci 12:728-730.

Petreanu L, Alvarez-Buylla A (2002) Maturation and death of adult-born olfactory bulb granule neurons: role of olfaction. J Neurosci 22:61066113.

Ramirez-Amaya V, Marrone DF, Gage FH, Worley PF, Barnes CA (2006) Integration of new neurons into functional neural networks. J Neurosci 26:12237-12241.

Rochefort C, Lledo PM (2005) Short-term survival of newborn neurons in the adult olfactory bulb after exposure to a complex odor environment. Eur J Neurosci 22:2863-2870.

Rochefort C, Gheusi G, Vincent JD, Lledo PM (2002) Enriched odor exposure increases the number of newborn neurons in the adult olfactory bulb and improves odor memory. J Neurosci 22:2679-2689.

Roullet F, Datiche F, Liénard F, Cattarelli M (2004) Cue valence representation studied by Fos immunocytochemistry after acquisition of a discrimination learning task. Brain Res Bull 64:31-38.

Sallaz M, Jourdan F (1996) Odour-induced c-fos expression in the rat olfactory bulb: involvement of centrifugal afferents. Brain Res 721:66-75.

Slotnick B (2001) Animal cognition and the rat olfactory system. Trends Cogn Sci 5:216-222.

Sultan S, Mandairon N, Kermen F, Garcia S, Sacquet J, Didier A (2010) Learning-dependent neurogenesis in the olfactory bulb determines longterm olfactory memory. FASEB J 24:2355-2363.

Tashiro A, Makino H, Gage FH (2007) Experience-specific functional modification of the dentate gyrus through adult neurogenesis: a critical period during an immature stage. J Neurosci 27:3252-3259.

Trouche S, Bontempi B, Roullet P, Rampon C (2009) Recruitment of adultgenerated neurons into functional hippocampal networks contributes to updating and strengthening of spatial memory. Proc Natl Acad Sci U S A 106:5919-5924.

Veyrac A, Sacquet J, Nguyen V, Marien M, Jourdan F, Didier A (2009) Novelty determines the effects of olfactory enrichment on memory and neurogenesis through noradrenergic mechanisms. Neuropsychopharmacology 34:786-795.

Winner B, Cooper-Kuhn CM, Aigner R, Winkler J, Kuhn HG (2002) Longterm survival and cell death of newly generated neurons in the adult rat olfactory bulb. Eur J Neurosci 16:1681-1689.

Yamaguchi M, Mori K (2005) Critical period for sensory experiencedependent survival of newly generated granule cells in the adult mouse olfactory bulb. Proc Natl Acad Sci U S A 102:9697-9702. 